\title{
Regenerated distributed Bragg reflector fiber lasers for high-temperature operation
}

\author{
Rongzhang Chen, ${ }^{1}$ Aidong Yan, ${ }^{1}$ Mingshan Li, ${ }^{1}$ Tong Chen, ${ }^{1}$ Qingqing Wang, ${ }^{1}$ \\ John Canning, ${ }^{2}$ Kevin Cook, ${ }^{2}$ and Kevin P. Chen ${ }^{1, *}$ \\ ${ }^{1}$ Department of Electrical and Computer Engineering, University of Pittsburgh, Pittsburgh, Pennsylvania 15213, USA \\ ${ }^{2}$ Interdisciplinary Photonics Laboratories, School of Chemistry, University of Sydney, NSW 1430, Australia \\ *Corresponding author: kchen@engr.pitt.edu
}

Received April 22, 2013; revised June 13, 2013; accepted June 13, 2013; posted June 14, 2013 (Doc. ID 188492); published July 10, 2013

\begin{abstract}
This Letter presents distributed Bragg reflector (DBR) fiber lasers for high-temperature operation at $750^{\circ} \mathrm{C}$. Thermally regenerated fiber gratings were used as the feedback elements to construct an erbium-doped DBR fiber laser. The output power of the fiber laser can reach $1 \mathrm{~mW}$ at all operating temperatures. The output power fluctuation tested at $750^{\circ} \mathrm{C}$ was $1.06 \%$ over a period of 7 hours. The thermal regeneration grating fabrication process opens new possibilities to design and to implement fiber laser sensors for extreme environments. (C) 2013 Optical Society of America

OCIS codes: (060.3510) Lasers, fiber; (060.3735) Fiber Bragg gratings; (060.2370) Fiber optics sensors; (120.6780) Temperature.
\end{abstract}

http://dx.doi.org/10.1364/OL.38.002490

Fiber lasers are powerful tools for sensing applications. Compared with passive fiber Bragg gratings, active sensing schemes based on fiber lasers normally show superior performance in terms of sensitivity, wavelength resolution, and accuracy. Recent research efforts [믈 have extended the operating temperature of fiber lasers to $600^{\circ} \mathrm{C}$ for high-temperature harsh environments. This is well beyond those prescribed for classic applications such as telecommunications. The successful development of all-fiber high-temperature fiber lasers relies on high-temperature-resistant fiber Bragg reflectors (FBGs). In view of those requirements, FBGs inscribed by ultrafast lasers, in the first place, have been proved to be promising candidates. For example, femtosecond laser inscribed Type II gratings were successfully used in fiber lasers operating at temperature up to $600^{\circ} \mathrm{C}$ [1] and output power scaling to $103 \mathrm{~W}$ [4], thanks to their high-temperature stability and subsequent power handling capability. Conventional UV-inscribed Type I fiber gratings are typically less temperature resistant than those fabricated by femtosecond lasers. Annealed UVinscribed fiber gratings in photosensitive fibers, with much simpler fabrication process and less costs, can sustain lasing up to $400^{\circ} \mathrm{C}$ [2].

In comparison with the gratings used in previously discussed fiber lasers, thermally regenerated gratings [5-7] are another class of high-temperature-resistant fiber gratings. The thermal regeneration of high-temperatureresistant gratings involves a mechanical relaxation process [] ], which is independent from the chemical composition of fibers and hydrogen loading processes. Thus, thermally regenerated gratings can be fabricated in a wide variety of fibers at low cost for extreme environments. Wang et al. has reported linear temperature and strain responses in thermally regenerated gratings from room temperature to $1100^{\circ} \mathrm{C}$ [9], which render regenerated gratings as a significant enhancement to conventional fiber grating applications, especially for extreme environments. Recently, we have demonstrated a regenerated-grating-based high-temperature pressure sensor, with which hydrostatic pressure $15-2400$ psi and high temperature $\left(24^{\circ} \mathrm{C}-800^{\circ} \mathrm{C}\right)$ can be accurately measured simultaneously [10].

In addition to passive sensing applications, regenerated gratings can offer considerable potential for applications in high-power laser [4] and active fiber sensing areas, where internal temperatures of fiber lasers can anneal conventional gratings out under high laser output power. With specially optimized optical fibers and strong seeds, regenerated gratings with $98.5 \%$ reflectance $(\sim 18 \mathrm{~dB})$ can be readily fabricated [11], which is normally sufficient for laser oscillation with a relatively small cavity size.

In this Letter, we present studies and characterization of high-temperature stable distributed Bragg reflector (DBR) fiber lasers using the regenerative grating technique. Two regenerated gratings are used as reflectors in a DBR fiber laser for high-temperature operation. The laser performance was characterized from the room temperature to $750^{\circ} \mathrm{C}$. Continuous monitoring for the laser output power was performed to quantify its stability at elevated temperatures. The regenerated gratings and output power of the laser are shown to be stable at $750^{\circ} \mathrm{C}$ for more than 7 hours. For fiber lasers demanding high-temperature stability or high-power operation, regenerated-grating-based DBR fiber laser (RDBR) stands out as a simple and reliable solution.

In this work, seed gratings were fabricated in standard telecommunications fibers (Corning SMF-28). To enhance photosensitivity, the fibers were soaked in a hydrogen chamber at $T \sim 25^{\circ} \mathrm{C}, P \sim 2400 \mathrm{psi}$ (165 bars) for about 1 week. Two $L=2.5 \mathrm{~cm}$ long FBGs with $\lambda_{\text {Bragg }} \sim 1550 \mathrm{~nm}$ were inscribed into the fiber core using a $248 \mathrm{~nm} \mathrm{KrF}$ laser and a phase mask. A cumulative fluence of $f_{\text {cum }}=1.32 \mathrm{~kJ} / \mathrm{cm}^{2}$ was used to produce highly saturated gratings with pulse fluence $f_{\text {pulse }} \sim 50 \mathrm{~mJ} / \mathrm{cm}^{2}$ and laser repetition rate $20 \mathrm{~Hz}$. The spectra of two seed gratings with matched resonant wavelengths are shown in Fig. 1. Excessive UV exposures induced $\Delta n_{\mathrm{DC}}>10^{-3}$ index change in the fiber core and index modulations 


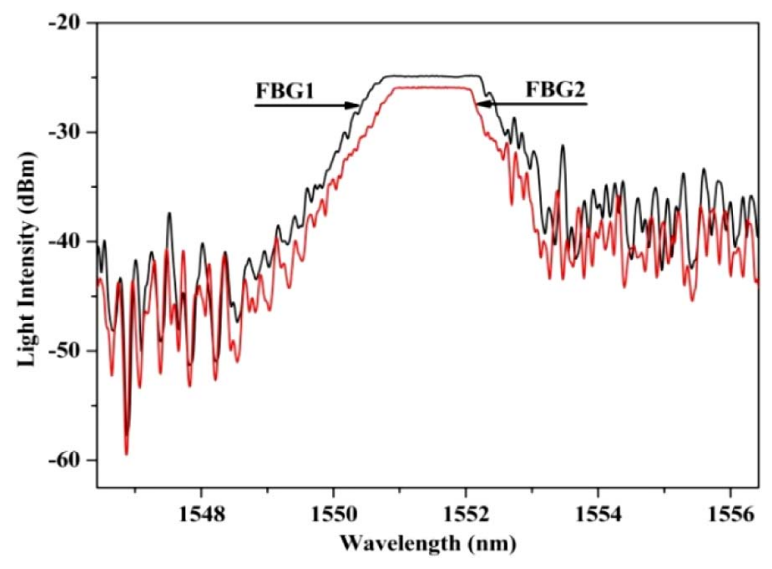

Fig. 1. Reflection spectra of the two matched fiber Bragg gratings.

were estimated to be $\Delta n_{1}=0.0015$ and $\Delta n_{2}=0.0013$ for the two gratings FBG1 and FBG2, respectively, shown in Fig. 1 .

Two matched gratings were fusion spliced to both ends of a $15 \mathrm{~cm}$ long erbium-doped fiber with absorption of $57.0 \mathrm{~dB} / \mathrm{m}$ at $980 \mathrm{~nm}$ (ER110-4/125 by nLight). The FBG2 in Fig. 1 with relatively weaker index modulation was used as the output coupler for the laser cavity. The spliced fiber was then post-hydrogen-loaded [8] in the hydrogen chamber for $t=\sim 2$ days before the regeneration process. After being removed from the hydrogen chamber, the fiber laser was immediately taken to a box furnace for thermal treatment. The temperature of the furnace was isochronally raised up to $T=800^{\circ} \mathrm{C}$ in $t \sim 2.5 \mathrm{~h}$. Seed gratings were then annealed at $T=$ $800^{\circ} \mathrm{C}$ for $t>50 \mathrm{~h}$. The evolution of the grating spectrum is shown in Fig. 2, and the changes for $\lambda_{\text {Bragg }}$ and reflection are shown in Fig. 3 . It is worth noting that the regeneration process of the two gratings was measured together. Thus, both of the gratings contributed to the reflection and the curve of peak wavelength shown in Fig. 3. There are five steps involved in the regeneration process. During Step A labeled in Fig. 3, the temperature of the box furnace isochronally increased at the rate of $5^{\circ} \mathrm{C} / \mathrm{min}$. A slow growth in reflection was initially

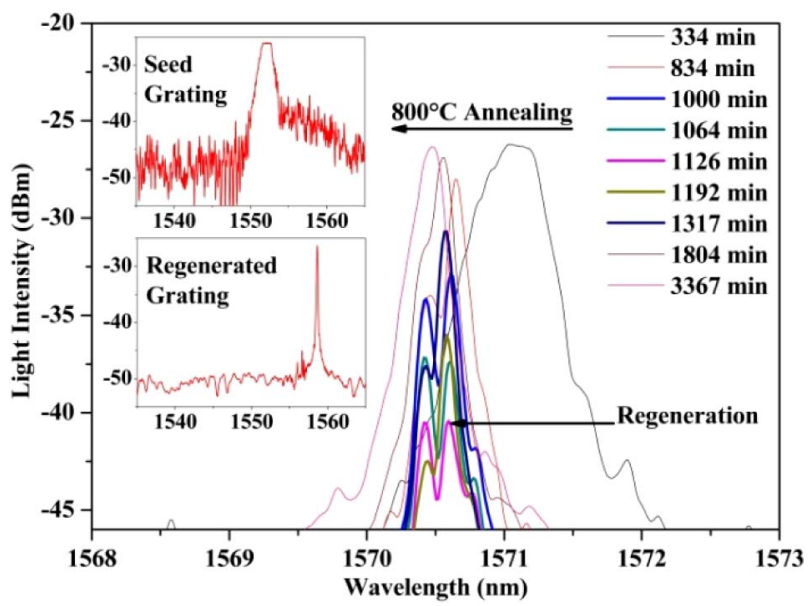

Fig. 2. Evolution of grating reflection spectra. Insets, reflection spectra of the seed at room temperature $\left(T=26^{\circ} \mathrm{C}\right)$ and regenerated grating at $800^{\circ} \mathrm{C}$.

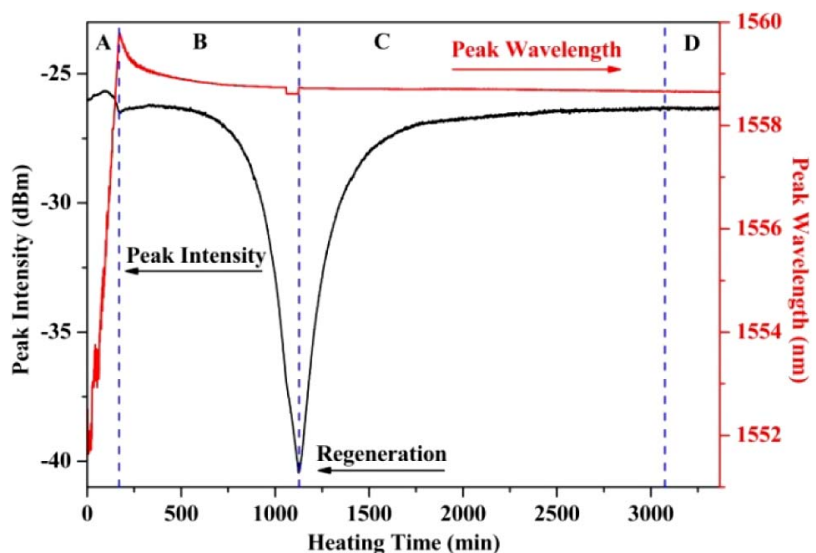

Fig. 3. Changes of grating strength and resonate wavelength during the regeneration process.

observed. This is mainly due to the degraded absorption of erbium-doped fiber [12]. When the fiber temperature was raised to $T=800^{\circ} \mathrm{C}$, the isothermal annealing started. The erasure of the seed gratings occurred during this period (labeled as Step B). This was followed by grating regeneration labeled as Step C. The strength of the regenerated gratings increased steadily during this period. A blue shift in $\lambda_{\text {Bragg }}$ was also observed in Step $\mathrm{B}$ and $\mathrm{C}$, which is attributed to relaxation of UV-induced $\Delta n_{\mathrm{DC}}$. At the early stage of the regeneration, two reflection peaks were on site as shown in Fig. 2. It is probably derived from the slightly different regeneration paces of FBG1 and FBG2, respectively. Peak reflectivity of regenerated FBGs increased and became stabilized after $t \sim 32 \mathrm{~h}$. The double-peak features disappeared once both FBGs were stabilized. In the last step, the regenerated FBGs were allowed to stabilize for another 5 hours until the FBG wavelength fluctuation was below the minimal resolution of the optical spectrum analyzer (OSA, Agilent 86140B with $70 \mathrm{pm}$ resolution) and the fluctuation of FBG strength was also less than $0.01 \mathrm{~dB} / \mathrm{h}$ at $T=800^{\circ} \mathrm{C}$. The index modulation of the regenerated grating FBG2 in Fig. 1 was estimated to be $\Delta n_{\text {reg }}=$

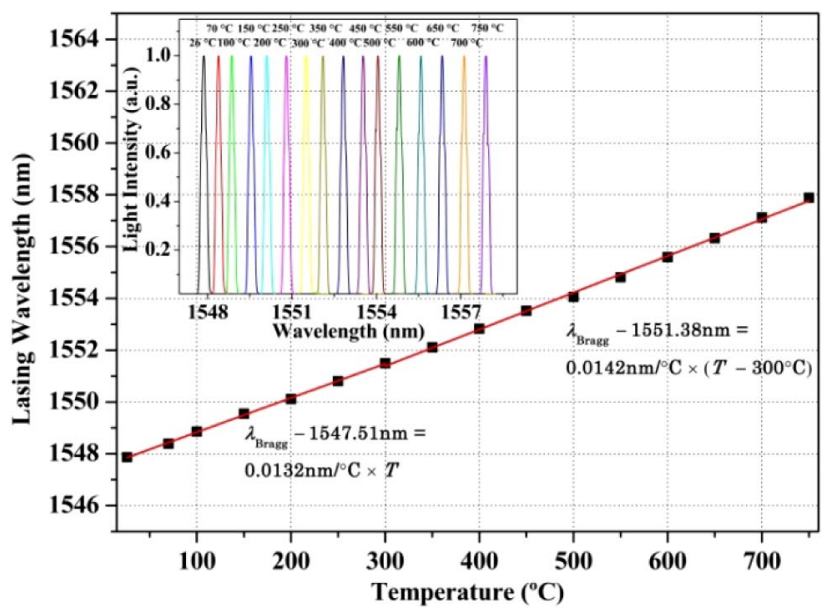

Fig. 4. Thermal wavelength shift of the fiber laser output (square) and its piecewise linear fitting curves (red). Inset, the normalized laser spectra under different temperatures. 


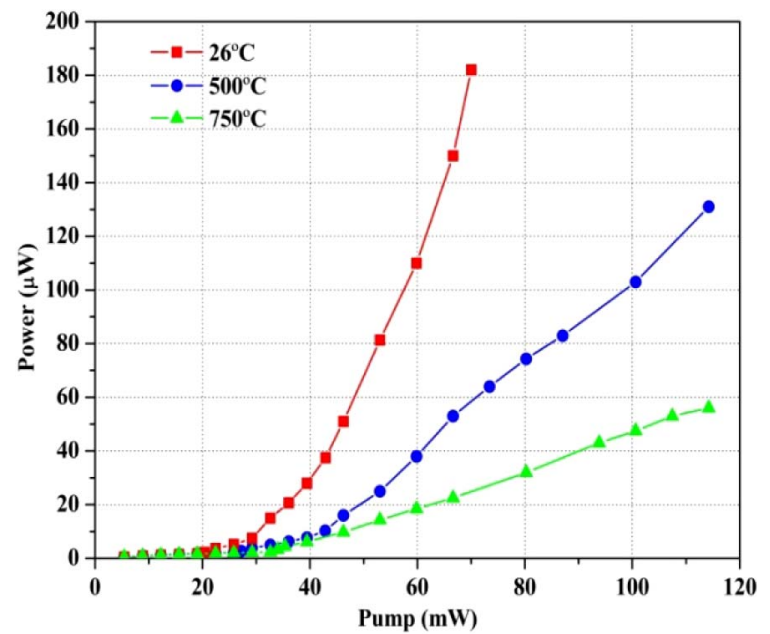

Fig. 5. Fiber laser output versus $980 \mathrm{~nm}$ pump power at three different temperatures.

$0.36 \times 10^{-4}$, resulting in more than $10 \mathrm{~dB}$ rejection in transmission with a FWHM bandwidth of $130 \mathrm{pm}$.

After the thermal regeneration process, the lasing characterization of the fiber laser was performed in a box furnace. A $980 \mathrm{~nm}$ diode laser, a wavelength division multiplexer, and the OSA were used for optical pumping and measurements, respectively. The laser performance was characterized between room temperature $(T=$ $26^{\circ} \mathrm{C}$ ) and $750^{\circ} \mathrm{C}$, which is $50^{\circ} \mathrm{C}$ below the regeneration temperature. Figure $\underline{4}$ presents lasing wavelength dependence on temperatures. Two linear wavelengthtemperature coefficients are obtained in respective temperature regimes. In the low temperature range from $26^{\circ} \mathrm{C}-300^{\circ} \mathrm{C}, \lambda_{\text {Bragg }}$ shifted linearly as a function of temperature where $\partial \lambda / \partial T \sim 0.0132 \mathrm{~nm} /{ }^{\circ} \mathrm{C}$. Above $300^{\circ} \mathrm{C}$ in the high temperature range, the coefficient increases to $\partial \lambda / \partial T \sim 0.0142 \mathrm{~nm} /{ }^{\circ} \mathrm{C}$. This changing temperature dependence in low and high temperature regimes is very similar to that of regenerated gratings [9].

The laser performance was further studied by quantifying the slope efficiency under different temperatures. Measurements of laser output versus pump power curves are shown in Fig. 5. At room temperature $T=26^{\circ} \mathrm{C}$, the laser exhibits the slope efficiency of $\eta_{s}=0.46 \%$. When the temperature was raised to $T=500^{\circ} \mathrm{C}$, the efficiency decreased to $\eta_{s}=0.175 \%$, due to the degraded gain spectrum, where the gain is depleted with increasing temperature by changing the saturated population inversion [12]. At the highest temperature $T=750^{\circ} \mathrm{C}$, the laser efficiency was significantly reduced to $\eta_{s}=0.069 \%$. The lasing threshold also increases due to the gain degradation at high temperatures. The threshold increased from $P_{\text {th }}=20 \mathrm{~mW}$ at the room temperature $T=26^{\circ} \mathrm{C}$ to $P_{\text {th }}=$ $27 \mathrm{~mW}$ at $T=500^{\circ} \mathrm{C}$ and finally $P_{\text {th }}=34 \mathrm{~mW}$ at $T=750^{\circ} \mathrm{C}$. Despite the gain degradation, the output power of the laser can reach $P_{\text {out }}>1 \mathrm{~mW}$ at all test temperatures.

To study high-temperature performance and stability of the fiber laser, several heating and cooling cycles between $26^{\circ} \mathrm{C}$ and $750^{\circ} \mathrm{C}$ were carried out for a period over 120 hours. No decay was observed in the grating strength or the lasing characteristics. Figure 6 shows the stability characteristics of the laser output power during a period

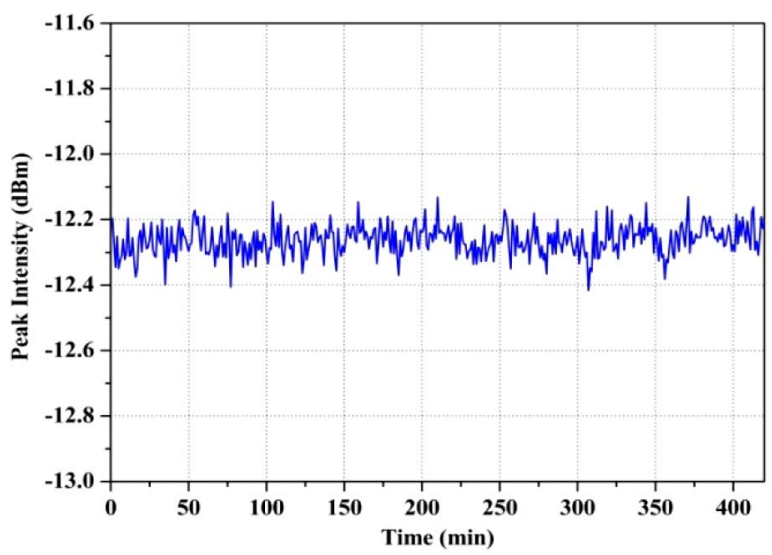

Fig. 6. Measured fiber laser output power over 7 hours at $750^{\circ} \mathrm{C}$.

of 7 hours of isothermal heating at $T=750^{\circ} \mathrm{C}$. The optical pump for the fiber laser was set at $P_{\text {pump }}=67 \mathrm{~mW}$. The OSA was used to measure the fiber laser output every minute. The total output power fluctuation shown in Fig. $\underline{6}$ is $1.06 \%$ with a $60 \mu \mathrm{W}$ output power.

In summary, we have applied the thermal regeneration process, a low-cost fiber Bragg grating fabrication technique, to produce an erbium-doped DBR (or RDBR) fiber laser for high-temperature environment operation up to $750^{\circ} \mathrm{C}$. Despite a long-fiber laser cavity, the RDBR fiber laser maintained stable operation at $750^{\circ} \mathrm{C}$ for over 7 hours with no performance degradation observed. The index modulation of the regenerated grating observed in this work might also be sufficient to produce a distributed feedback laser with much better longitudinal mode stability. The thermal regeneration FBG fabrication process opens new possibilities to design and implement fiber laser sensors for extreme environments.

This work was supported by the National Science Foundation (CMMI-0644681 and CMMI-1054652) and the Department of Energy (DE-FE0003859).

\section{References}

1. Y. Lai, A. Martinez, I. Khrushchev, and I. Bennion, Opt. Lett. 31, 1672 (2006).

2. Y. Shen, Y. Qiu, B. Wu, W. Zhao, S. Chen, T. Sun, and K. T. V. Grattan, Opt. Express 15, 363 (2007).

3. B.-O. Guan, Y. Zhang, H.-J. Wang, D. Chen, and H.-Y. Tam, Opt. Express 16, 2958 (2008).

4. N. Jovanovic, M. Åslund, A. Fuerbach, S. D. Jackson, G. D. Marshall, and M. J. Whitford, Opt. Lett. 32, 2804 (2007).

5. Z. Bowei and K. Mojtaba, IEEE Sens. J. 7, 586 (2007).

6. S. Bandyopadhyay, J. Canning, M. Stevenson, and K. Cook, Opt. Lett. 33, 1917 (2008).

7. S. Bandyopadhyay, J. Canning, P. Biswas, M. Stevenson, and K. Dasgupta, Opt. Express 19, 1198 (2011).

8. E. Lindner, J. Canning, C. Chojetzki, S. Brückner, M. Becker, M. Rothhardt, and H. Bartelt, Appl. Opt. 50, 2519 (2011).

9. T. Wang, L.-Y. Shao, J. Canning, and K. Cook, Opt. Lett. 38, 247 (2013).

10. T. Chen, R. Chen, C. Jewart, B. Zhang, K. Cook, J. Canning, and K. P. Chen, Opt. Lett. 36, 3542 (2011).

11. J. Canning, M. Stevenson, J. Fenton, M. Aslund, and S. Bandyopadhyay, Proc. SPIE 7503, 750326 (2009).

12. N. Kagi, A. Oyobe, and K. Nakamura, J. Lightwave Technol. 9, 261 (1991). 